Copyright@2006 IEEE. Reprinted from:

T. Roman, S. Visuri and V. Koivunen. Blind frequency synchronization in OFDM via diagonality criterion. To appear in IEEE Transactions on Signal Processing.

This material is posted here with permission of the IEEE. Such permission of the IEEE does not in any way imply IEEE endorsement of any of Helsinki University of Technology's products or services. Internal or personal use of this material is permitted. However, permission to reprint/republish this material for advertising or promotional purposes or for creating new collective works for resale or redistribution must be obtained from the IEEE by writing to pubs-permissions@ieee.org.

By choosing to view this document, you agree to all provisions of the copyright laws protecting it. 


\title{
Blind frequency synchronization in OFDM via diagonality criterion
}

\author{
Timo Roman, Samuli Visuri and Visa Koivunen Senior Member, IEEE
}

\begin{abstract}
In this paper we address the problem of blind carrier frequency offset (CFO) estimation in OFDM systems in the case of frequency selective channels. CFO destroys the orthogonality between the carriers leading to nondiagonal signal covariance matrices in frequency domain. The proposed blind method enforces a diagonal structure by minimizing the power of non-diagonal elements. Hence, the orthogonality property inherent to OFDM transmission with cyclic prefix is restored. The method is blind since it does not require a priori knowledge of the transmitted data or the channel, and does not need any virtual subcarriers. A closed-form solution is derived, which leads to accurate and computationally efficient CFO estimation in multipath fading environments. Consistency of the estimator is proved and the convergence rate as a function of the sample size is analyzed as well. To assess the large sample performance, we derive the Cramér-Rao bound (CRB) for the blind CFO estimation problem. The CRB is derived assuming a general Gaussian model for the OFDM signal, which may be applied to both circular and non-circular modulations. Finally, simulation results on CFO estimation are reported using a realistic channel model.
\end{abstract}

Index Terms-OFDM, frequency offset, blind methods, Cramér-Rao bound, covariance matrix.

\section{INTRODUCTION}

$\mathbf{O}$ RTHOGONAL Frequency Division Multiplexing (OFDM) transmission has already proven successful for both wireless (e.g. DVB-T and WLAN) and wireline applications (e.g. ADSL). Multicarrier modulation is a powerful technique to handle impairments of wireless communication media such as multipath propagation due to its ability to turn frequency selective channels into a set of narrowband frequency flat channels [1], [2]. Hence, OFDM is a viable candidate for future beyond $3 \mathrm{G}$ wireless communications standards.

One of the main drawbacks of OFDM over singlecarrier systems is its high sensitivity to carrier frequency offsets (CFO) caused by oscillator inaccuracies and Doppler shift due to mobility [3]. This gives rise to

Manuscript received March 10, 2005; revised Sept. 06, 2005. This work was supported in part by the Academy of Finland and GETA Graduate School.

T. Roman and V. Koivunen are with the SMARAD CoE, Signal Processing Laboratory, Department of Electrical Engineering, Helsinki University of Technology, FIN-02015 HUT, Finland (e-mail: \{Timo.Roman, Visa.Koivunen\}@hut.fi).

$\mathrm{S}$. Visuri is with the Radio Technologies laboratory, Nokia Research Center, P.O. Box 407, FI-00045 NOKIA GROUP, Finland (e-mail: Samuli.Visuri@nokia.com). inter-carrier interference (ICI) and reduction in amplitude for the desired subcarriers [2]. The issue is critical when higher data rates are required, and relatively large number of subcarriers together with very narrow guard bands are used [4], [5]. Imperfect CFO estimation has also a detrimental impact on channel estimation [6]. Consequently, frequency offset estimation and compensation must be accomplished with high fidelity.

Most of the existing CFO estimators for OFDM rely on periodically transmitted pilot symbols [7]-[10]. However, this takes place at the expense of bandwidth efficiency, especially in the case of continuous transmissions (e.g. DVB-T). Therefore, pilot-based schemes are mainly suited for packet-oriented applications.

Semi-blind approaches proposed in the literature aim at improving bandwidth efficiency [11]-[13]. Those usually rely on various assumptions such as the usage of a single pilot symbol, two identical consecutive OFDM data blocks [11], decision-directed processing [13], or some specific structure within the OFDM symbol [14].

Blind (non data-aided) methods have received considerable amount of attention over the past years. Among different classes of methods, one may distinguish subspacebased methods [15]-[17], which were recently shown to be equivalent to the maximum likelihood (ML) estimator [18]. Those methods rely on the low rank signal model induced by either some unmodulated carriers or virtual subcarriers (VSC). VSC are unmodulated subcarriers at the edges of the OFDM block which aim at minimizing the interference caused to adjacent OFDM systems. Their number is dictated by system design requirements [19]. While OFDM systems are suited by design to multipath transmission, many existing CFO estimators deal only with frequency flat channels [20], [21]. A ML estimator was proposed in [20] assuming an additive white Gaussian noise (AWGN) channel. Unfortunately, it is not robust to multipath propagation. Extension of ML methods to multipath Rayleigh fading channels may be found in [22]. More recently, non-circularity introduced by real-valued modulations (e.g., BPSK) was exploited in [23], [24]. Constant modulus (CM) constellations allow highly accurate $\mathrm{CFO}$ estimation also, see e.g. [25], [26]. An approach relying on a kurtosis type of criterion was proposed in [27]. Most of the CFO estimation algorithms in the literature exploit second-order cyclostationarity [20], [28], [29]. Cyclostationarity in the received signal is induced by the 
cyclic prefix (CP). In addition, higher-order statistics have been exploited in [30]. Joint frequency synchronization and channel estimation schemes have been proposed as well [31]. They typically require solving multidimensional optimization problems. Hence, their complexity limits their practical use.

In this paper, a blind method for CFO estimation and compensation in frequency selective channels is introduced. The method does not require a priori knowledge of the transmitted data or the multipath channel. Bandwidth efficiency remains high as no pilot or virtual carriers are needed. Information on the CFO is embedded in the received signal covariance matrix. Based on that property, a cost function may be derived, and the sample covariance matrix may be used to estimate the CFO in practice. The cost function minimizes the total off-diagonal power induced by ICI in frequency domain. Enforcing a diagonal structure aims at restoring the orthogonality of the transmission inherent to perfectly synchronized OFDM modulation. The proposed algorithm applies to both real (e.g., BPSK, PAM) and complex modulations (e.g., QPSK, 8PSK, 16QAM, 64QAM), and extends to asymmetric constellations as well. Hence it may be used in a wide range of OFDM transceivers. Furthermore, accurate CFO estimation is achieved at low SNR regime, where decisiondirected methods are most likely to fail.

A closed-form expression is found for the cost function which leads to low complexity and accurate computational solution. The proposed method is shown to yield a consistent estimate of the CFO. Convergence in the mean square is proved also, and the convergence rate of the algorithm as a function of the sample size is analyzed in a closedform as well.

In order to assess the efficiency of the estimator, we derive the Cramér-Rao bound (CRB) for blind CFO estimators. We model the OFDM signal as a Gaussian process. Depending on the modulation in use, the received signal may be complex circular (proper) or not [32]. Consequently, the CRB has to be derived for a general Gaussian model which includes both signal covariance and pseudo-covariance matrices [23], [33]. Simulation results for the presented estimator show a performance close to the CRB. Numerical results are also in par with the theoretical analysis of the convergence rate.

The rest of the paper is organized as follows. Section II introduces the system model. In Section III, second order statistics for real and complex random vectors are briefly reviewed. In Section IV, we define a cost function for the blind method and derive a closed-form expression for it. Then, a new blind frequency offset estimation algorithm is introduced. Consistency of the CFO estimator is proved in Section $\mathrm{V}$, and the convergence rate is analyzed as well. In Section VI, we derive the CRB for the blind CFO estimation problem. Simulation results are reported in Section VII, where we demonstrate the reliable performance of the proposed method at different noise levels for both fixed and random channel impulse responses. Finally, Section VIII concludes the paper. Proofs and additional derivations may be found in the Appendix.

The following notation is used throughout the paper:

\begin{tabular}{|c|c|}
\hline $\begin{array}{lr}a, \quad \mathbf{a}, & \mathbf{A} \\
\mathbf{A}^{T} & \mathbf{A}^{H}\end{array}$ & scalar $a$, column vector $\mathbf{a}, \quad$ matrix $\mathbf{A}$; \\
\hline $\mathbf{A}^{1 / 2}, \mathbf{A}^{-1}$ & Hermitian square-root of $\mathbf{A}$, invers \\
\hline$[\mathbf{A}]_{i j}$ & $(i, j)$ element of matrix $\mathbf{A}$ \\
\hline $\mathbf{I}_{N}$ & identity matrix of size $N \times N$ \\
\hline $\mathbb{1}_{N}$ & $N \times N$ matrix filled with ones; \\
\hline $\begin{array}{l}\mathbf{0}_{N \times N} \times N \\
\operatorname{diag}\{\mathbf{a}\}\end{array}$ & $N \times N$ matrix filled with zeros; \\
\hline unas $\{a$ & $\begin{array}{l}\mathbf{a}=\left[a_{1}, \ldots, a_{N}\right]^{T} \text { on the main diagonal; } \\
\text { expectation operator; }\end{array}$ \\
\hline $\begin{array}{l}\operatorname{Tr},{ }^{*} \\
\|\|_{F}\end{array}$ & $\begin{array}{l}\text { trace operator, elementwise conjugation; } \\
\text { Frobenius norm; }\end{array}$ \\
\hline $\begin{array}{ll}\otimes, & \odot \\
\propto, & \bmod N \\
\text { vec } & \end{array}$ & $\begin{array}{l}\text { Kronecker product, Hadamard product; } \\
\text { proportional to, modulo } N \text { operator; } \\
\text { stacks columns of a matrix on top of } \\
\text { each other; }\end{array}$ \\
\hline $\begin{array}{l}\text { Re, } \quad \mathrm{Im} \\
\arg \end{array}$ & $\begin{array}{l}\text { real part, imaginary part; } \\
\text { argument of a complex number; }\end{array}$ \\
\hline$\delta_{k, l}$ & $\delta_{k, l}=1$ if $k=l, \delta_{k, l}=0$ if $k \neq l$ \\
\hline i.i.d. & independent and identically distributed; \\
\hline & nvergence with probability one. \\
\hline
\end{tabular}

\section{SySTEM MODEL}

We use a general OFDM transmission model [16] (see Fig. 1). The $k$-th modulated OFDM block may be written as

$$
\mathbf{b}(k)=\mathbf{F}_{N} \mathbf{a}(k),
$$

where $\mathbf{F}_{N}=1 / \sqrt{N}\{\exp (j 2 \pi m n / N)\}_{m, n=0, \ldots, N-1}$ is the $N \times N$ inverse discrete Fourier transform (IDFT) matrix, $N$ is the number of subcarriers, and $\mathbf{a}(k)$ is the complex-valued $N \times 1$ symbol vector.

The received OFDM $N \times 1$ signal block in time domain after cyclic prefix removal, including frequency offset, may be expressed as [19]:

$$
\mathbf{r}_{\epsilon}(k)=e^{j 2 \pi \epsilon(k P+L) / N} \mathbf{C}(\epsilon) \widetilde{\mathbf{H}} \mathbf{b}(k)+\mathbf{w}(k),
$$

where:

- The length of the cyclic prefix is $L$ and the total OFDM block length is $P=N+L$.

- The matrix $\widetilde{\mathbf{H}}$ of size $N \times N$ is a circulant channel matrix [1], [19] built from the channel impulse vector $\mathbf{h}$ in time domain of size $L_{h} \times 1$, with $L_{h} \leq L$. Furthermore, the channel is assumed to be quasistationary over the observation period.

- The diagonal matrix $\mathbf{C}(\epsilon)$ of size $N \times N$ introduces the frequency offset $\epsilon$ :

$$
\mathbf{C}(\epsilon)=\operatorname{diag}\left\{e^{j \frac{2 \pi n \epsilon}{N}}, n=0, \ldots, N-1\right\} .
$$

- The noise term $\mathbf{w}$ in (2) is assumed to be circular complex Gaussian [32] with covariance matrix $\sigma^{2} \mathbf{I}_{N}$. 


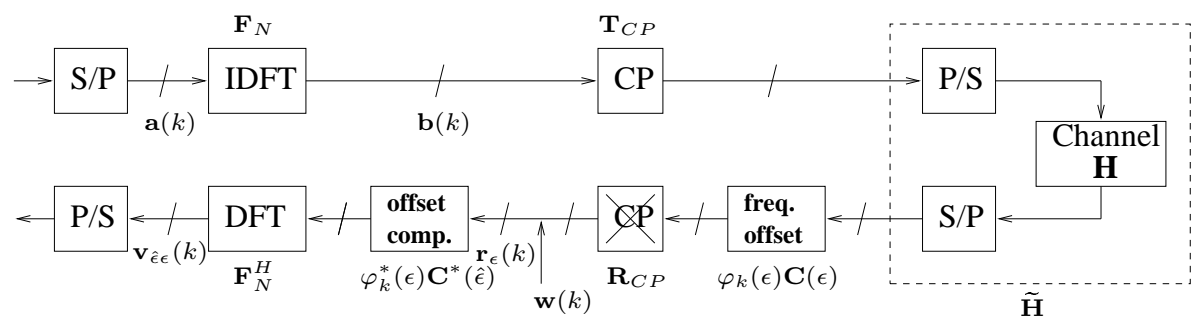

Fig. 1. OFDM transmission chain.

The signal and noise processes are assumed to be mutually independent, and i.i.d. over time index $k$.

The quantity $\epsilon$ is referred to as normalized frequency offset with respect to inter-carrier spacing. The effective frequency deviation is $\epsilon \Delta f[\mathrm{~Hz}]$, where $\Delta f=B / N$ is the inter-carrier spacing and $B[\mathrm{~Hz}]$ is the bandwidth allocated to the system. The proposed algorithm is aimed at estimating the fractional CFO, i.e., we assume that $\epsilon \in[0,1[$. Hence, it may track small CFO or the residual offset when the initially larger CFO has been significantly reduced by other means [7], [14], [18], [34].

In case of perfect frequency synchronization (i.e. $\epsilon=0$, $\left.\mathbf{C}(0)=\mathbf{I}_{N}\right)$, the received signal becomes

$$
\mathbf{r}(k)=\widetilde{\mathbf{H}} \mathbf{b}(k)+\mathbf{w}(k) .
$$

Given an estimate $\hat{\epsilon}$ of the true value $\epsilon$, CFO compensation may be performed in time domain at the receiver prior to the discrete Fourier transform. Based on (2), the resulting $\mathrm{CFO}$ compensated received vector, $\mathbf{v}_{\hat{\epsilon} \epsilon}$, may be expressed as

$$
\begin{aligned}
\mathbf{v}_{\hat{\epsilon} \epsilon}(k)= & \varphi_{k}^{*}(\hat{\epsilon}) \mathbf{F}_{N}^{H} \mathbf{C}^{*}(\hat{\epsilon}) \mathbf{r}_{\epsilon}(k) \\
= & \varphi_{k}^{*}(\hat{\epsilon}-\epsilon) \mathbf{F}_{N}^{H} \mathbf{C}^{*}(\hat{\epsilon}-\epsilon) \widetilde{\mathbf{H}} \mathbf{b}(k)+\ldots \\
& \ldots+\varphi_{k}^{*}(\hat{\epsilon}) \mathbf{F}_{N}^{H} \mathbf{C}^{*}(\hat{\epsilon}) \mathbf{w}(k),
\end{aligned}
$$

where $\mathbf{C}(\hat{\epsilon})$ and $\mathbf{C}(\hat{\epsilon}-\epsilon)$ possess the structure defined in (3) and $\varphi_{k}(\epsilon)=\exp (j 2 \pi \epsilon(k P+L) / N)$.

\section{SECOND ORDER STATISTICS FOR REAL AND COMPLEX MODULATIONS}

In order to fully characterize the second order statistics of a complex-valued random vector $\mathbf{u}$, two matrices are needed [33]:

$$
\begin{aligned}
\operatorname{cov}(\mathbf{u}) & \triangleq \mathrm{E}\left[(\mathbf{u}-\mathrm{E}[\mathbf{u}])(\mathbf{u}-\mathrm{E}[\mathbf{u}])^{H}\right] \\
\operatorname{pcov}(\mathbf{u}) & \triangleq \mathrm{E}\left[(\mathbf{u}-\mathrm{E}[\mathbf{u}])(\mathbf{u}-\mathrm{E}[\mathbf{u}])^{T}\right]
\end{aligned}
$$

where $\operatorname{cov}(\mathbf{u})$ is the covariance matrix of $\mathbf{u}$ and $\operatorname{pcov}(\mathbf{u})$ is the pseudo-covariance matrix of $\mathbf{u}$. The pseudocovariance matrix is sometimes also referred to as conjugate covariance matrix or complementary covariance matrix [32], [33]. A complex-valued random vector $\mathbf{u}$ is called circular (or proper [32]) random vector if its pseudo-covariance vanishes, i.e., $\operatorname{pcov}(\mathbf{u})=\mathbf{0}_{N \times N}$.
Depending whether complex or real symbol modulations are used, the received signal becomes complex circular or non-circular. More specifically, let us assume i.i.d. data bits, which are mapped onto the complex zero mean data vector $\mathbf{a}(k)$. From now on, we drop the time index $k$ for simplicity. Let us denote the covariance and pseudo-covariance matrices of $\mathbf{a}$ by $\mathbf{Q}_{\mathbf{a}}=\operatorname{cov}(\mathbf{a})$ and $\mathbf{P}_{\mathbf{a}}=\operatorname{pcov}(\mathbf{a})$, respectively. For instance, with BPSK symbols, both $\mathbf{Q}_{\mathbf{a}}$ and $\mathbf{P}_{\mathbf{a}}$ are non-zero. With QAM symbols, $\mathbf{P}_{\mathbf{a}}=\mathbf{0}_{N \times N}$, since real and imaginary parts of a are independent. Hence, complete second order statistics include both signal covariance and pseudo-covariance matrices [33].

\section{BLIND CFO ESTIMATION IN OFDM VIA DIAGONALITY CRITERION}

\section{A. Signal covariance matrix}

In this section, we introduce a carrier frequency offset (CFO) estimation method for OFDM based on the signal covariance matrix. Hence, it applies to both real- and complex-valued modulations. This derivation extends the preliminary results presented in [29]. Without loss of generality, we assume that unit energy QPSK constellation is used. Hence, the symbol vector covariance matrix is $\operatorname{cov}(\mathbf{a})=\mathbf{I}_{N}$. However, even though the QPSK case is considered only, results presented in this paper extend to any other kind of real- or complex-valued modulation scheme, e.g., BPSK, 8PSK, 16QAM, 64QAM. From the transmission equation (4), $\mathbf{Q}=\operatorname{cov}(\mathbf{r})$ may expressed as

$$
\begin{aligned}
\mathbf{Q} & =\mathrm{E}\left[(\widetilde{\mathbf{H}} \mathbf{b}+\mathbf{w})(\widetilde{\mathbf{H}} \mathbf{b}+\mathbf{w})^{H}\right] \\
& =\widetilde{\mathbf{H}} \mathbf{F}_{N} \operatorname{cov}(\mathbf{a}) \mathbf{F}_{N}^{H} \widetilde{\mathbf{H}}^{H}+\operatorname{cov}(\mathbf{w}) \\
& =\widetilde{\mathbf{H}} \widetilde{\mathbf{H}}^{H}+\sigma^{2} \mathbf{I}_{N},
\end{aligned}
$$

where (10) follows from the independence between the zero-mean noise and data processes. From (10) to (11), we used $\operatorname{cov}(\mathbf{a})=\mathbf{I}_{N}$, the unitary property of the IDFT matrix, and the fact that $\operatorname{cov}(\mathbf{w})=\sigma^{2} \mathbf{I}_{N}$.

\section{B. Cost function minimizing the total off-diagonal power}

Let $\widetilde{\mathbf{Q}}_{k}(\mu)=\operatorname{cov}\left(\mathbf{v}_{\mu \epsilon}(k)\right)$, where $\mathbf{v}_{\mu \epsilon}$ denotes $\mathbf{v}_{\hat{\epsilon} \epsilon}$ in (5) evaluated at $\hat{\epsilon}=\mu$. Then $\widetilde{\mathbf{Q}}_{k}(\mu)$ and $\mathbf{Q}$ are related 
by

$$
\begin{aligned}
\widetilde{\mathbf{Q}}_{k}(\mu) & =\mathbf{F}_{N}^{H} \mathbf{C}^{*}(\mu-\epsilon) \widetilde{\mathbf{H}} \widetilde{\mathbf{H}}^{H} \mathbf{C}(\mu-\epsilon) \mathbf{F}_{N}+\sigma^{2} \mathbf{I}_{N}{ }^{1} \\
& =\mathbf{F}_{N}^{H} \mathbf{C}^{*}(\mu-\epsilon) \mathbf{Q} \mathbf{C}(\mu-\epsilon) \mathbf{F}_{N} \\
& \triangleq \widetilde{\mathbf{Q}}(\mu)
\end{aligned}
$$

where we followed the same intermediate steps and assumptions as for equations (9)-(11).

Null or perfectly compensated frequency offset $(\mu=\epsilon)$ leads to a perfectly orthogonal transmission, and $\widetilde{\mathbf{Q}}(\mu)$ becomes diagonal. Proof is straightforward and may be found in Appendix I. Off-diagonal elements are introduced by inter-carrier interference and should be minimized. Hence, for a given offset compensation value $\mu$, we choose the total off-diagonal power $\mathcal{J}(\mu)$ of $\widetilde{\mathbf{Q}}(\mu)$ as a cost function to be minimized:

$$
\mathcal{J}(\mu)=\left\|\widetilde{\mathbf{Q}}(\mu) \odot\left(\mathbb{1}_{N}-\mathbf{I}_{N}\right)\right\|_{F}^{2}, \quad \mu \in[0,1[,
$$

where $\odot$ stands for the Hadamard product and $\mathbb{1}_{N}$ denotes a $N \times N$ matrix of 1's. Notice that the noise does not have any influence in theory provided that its covariance matrix is diagonal, i.e., it is uncorrelated.

In order to guarantee the identifiability of the CFO, the channel covariance matrix $\widetilde{\mathbf{H}} \widetilde{\mathbf{H}}^{H}$ should have offdiagonal terms ${ }^{2}$. Otherwise, the matrix $\mathbf{Q}$ is diagonal and $\mathbf{C}(\epsilon) \mathbf{Q} \mathbf{C}^{*}(\epsilon)$ does not contain information on $\epsilon$ anymore.

Now, Theorem 1 proves the existence of a unique minimum of $\mathcal{J}$ at $\epsilon$ in $[0,1[$.

Theorem 1: Let $\mathcal{J}: \mu \mapsto \mathcal{J}(\mu)$ be defined as in (15). Then, assuming a non-diagonal channel covariance matrix $\widetilde{\mathbf{H}} \widetilde{\mathbf{H}}^{H}$,

1) $\mathcal{J}(\epsilon)=0$.

2) $\mathcal{J}(\mu)>0, \forall \mu \neq \epsilon, \mu \in[0,1[$.

Proof is given in Appendix I. Hence, true CFO may be found by driving $\mathcal{J}(\mu)$ to zero. In practice, only an estimate $\widehat{\widetilde{\mathbf{Q}}}_{K}(\mu)$ of $\widetilde{\mathbf{Q}}(\mu)$ is available (subscript $K$ refers to the sample size). Then, an estimate $\hat{\epsilon}$ of the CFO $\epsilon$ may found by

$$
\hat{\epsilon}_{K}=\arg \min _{\mu \in[0,1[} \widehat{\mathcal{J}}_{K}(\mu)
$$

where the estimated cost function $\widehat{\mathcal{J}}_{K}$ after $K$ received OFDM blocks is given as

$$
\widehat{\mathcal{J}}_{K}(\mu)=\left\|\widehat{\widetilde{\mathbf{Q}}}_{K}(\mu) \odot\left(\mathbb{1}_{N}-\mathbf{I}_{N}\right)\right\|_{F}^{2}, \quad \mu \in[0,1[.
$$

The cost function $\widehat{\mathcal{J}}_{K}$ penalizes the off-diagonal energy of $\widehat{\widetilde{\mathbf{Q}}}_{K}$. It measures the loss of orthogonality due to CFO.

\footnotetext{
${ }^{1}$ The block dependent phase term $\varphi_{k}(\epsilon)$ in (2) cancels out in the derivation of $\widetilde{\mathbf{Q}}_{k}(\mu)=\operatorname{cov}\left(\mathbf{v}_{\mu \epsilon}(k)\right)$. Hence $\widetilde{\mathbf{Q}}_{k}(\mu)$ does not depend on the block index $k$ and will be further denoted by $\widetilde{\mathbf{Q}}(\mu)$.

${ }^{2}$ The channel is required to be multipath, i.e., it has at least two nonzero taps in time domain. The latter assumption is fulfilled in practical OFDM transmissions.
}

The proposed method is blind in a sense that minimization of $\hat{\mathcal{J}}_{K}$ may be performed without any knowledge of the wireless channel $\widetilde{\mathbf{H}}$ or pilot symbols.

Note that exploiting the pseudo-covariance matrix alone instead of the covariance is not a viable choice here. In theory, $\widetilde{\mathbf{P}}_{k}(\mu)=\operatorname{pcov}\left(\mathbf{v}_{\mu \epsilon}(k)\right)$ contains information on the CFO for real-valued modulations. However, the block dependent phase term $\varphi_{k}(\epsilon)$ in (2) drives its sample estimate to zero for large sample sizes.

\section{Closed-form expression for the cost function}

In order to find a computationally efficient way to estimate the CFO, a closed form expression of the cost function is derived next. To begin with, let us state the following useful lemma:

Lemma 1: Given any non-zero matrix $\mathbf{R}$ of size $N \times N$, and matrices $\mathbf{F}_{N}$ and $\mathbf{C}(\mu)$ as defined in (1) and (3), respectively, the function $\mathcal{K}_{\mathbf{R}}$ of the real parameter $\mu$ given by

$$
\mathcal{K}_{\mathbf{R}}(\mu)=\left\|\left(\mathbf{F}_{N}^{H} \mathbf{C}^{*}(\mu) \mathbf{R C}(\mu) \mathbf{F}_{N}\right) \odot\left(\mathbb{1}_{N}-\mathbf{I}_{N}\right)\right\|_{F}^{2}
$$

can be written as $\mathcal{K}_{\mathbf{R}}(\mu)=a+b \cos (2 \pi \mu)+c \sin (2 \pi \mu)$, where scalars $a, b, c \in \mathbb{R}$ are specific to the matrix $\mathbf{R}$.

Proof of Lemma 1 as well as expressions for $a, b$ and $c$ are given in Appendix II.

Let us define $\mathbf{R}=\mathbf{C}(\epsilon) \widehat{\mathbf{Q}}_{K} \mathbf{C}^{*}(\epsilon)$, where $\widehat{\mathbf{Q}}_{K}$ is the sample covariance matrix for $\epsilon=0$ (see (24)-(25)). Then, $\widehat{\widetilde{\mathbf{Q}}}_{K}(\mu)=\mathbf{F}_{N}^{H} \mathbf{C}^{*}(\mu) \mathbf{R C}(\mu) \mathbf{F}_{N}$, and by applying Lemma 1 , the estimate $\widehat{\mathcal{J}}_{K}$ of the cost function in (17) may be expressed as

$$
\widehat{\mathcal{J}}_{K}(\mu)=a+b \cos (2 \pi \mu)+c \sin (2 \pi \mu),
$$

where $a, b, c \in \mathbb{R}$. From (18), $\widehat{\mathcal{J}}_{K}$ is obviously a periodic function of $\mu$ with period 1 . Hence, we may further restrict our analysis to the interval $[0,1[$. An example of the cost function is depicted in Figure 2, at $15 \mathrm{~dB}$ SNR and with $K=200$ observed blocks. The minimum is reached at $\mu=0.4379$, while the true offset is $\epsilon=0.43$. The sinusoidal form may be clearly observed, which is in par with the result in (18).

Differentiating $\widehat{\mathcal{J}}_{K}$ with respect to $\mu$ yields

$$
\frac{\partial}{\partial \mu} \widehat{\mathcal{J}}_{K}(\mu)=-2 \pi b \sin (2 \pi \mu)+2 \pi c \cos (2 \pi \mu) \text {. }
$$

The extrema of $\widehat{\mathcal{J}}_{K}$ in $[0,1$ are found in closed form as

$$
\hat{\nu}_{K_{i}}=\frac{1}{2 \pi} \arctan \left\{\frac{c}{b}\right\}+\frac{i}{2} \bmod 1, \quad i=0,1 .
$$

Given the form in (18), it is sufficient to evaluate $\widehat{\mathcal{J}}_{K}$ at three points in order to solve for $a, b$ and $c$. We choose the following equi-spaced points ${ }^{3} 0,1 / 3$ and $2 / 3$ within

\footnotetext{
${ }^{3} \mathrm{~A}$ similar minimization/maximization procedure in closed-form was employed in [27].
} 
the interval $\left[0,1\left[\right.\right.$. Hence, the extrema of $\widehat{\mathcal{J}}_{K}$ may be given in closed form by

$$
\begin{aligned}
\hat{\nu}_{K_{i}}= & \frac{1}{2 \pi} \arctan \left\{\frac{\sqrt{3}\left(\widehat{\mathcal{J}}_{K}\left(\frac{1}{3}\right)-\widehat{\mathcal{J}}_{K}\left(\frac{2}{3}\right)\right)}{2 \widehat{\mathcal{J}}_{K}(0)-\widehat{\mathcal{J}}_{K}\left(\frac{1}{3}\right)-\widehat{\mathcal{J}}_{K}\left(\frac{2}{3}\right)}\right\}+\ldots \\
& \ldots+\frac{i}{2} \quad \bmod 1, \quad i=0,1 .
\end{aligned}
$$

Finally, the frequency offset estimate is found by choosing the value corresponding to the minimum of the cost function

$$
\hat{\epsilon}_{K}= \begin{cases}\hat{\nu}_{K_{0}} & \text { if } \widehat{\mathcal{J}}_{K}\left(\hat{\nu}_{K_{0}}\right)<\widehat{\mathcal{J}}_{K}\left(\hat{\nu}_{K_{1}}\right) \\ \hat{\nu}_{K_{1}} & \text { otherwise. }\end{cases}
$$

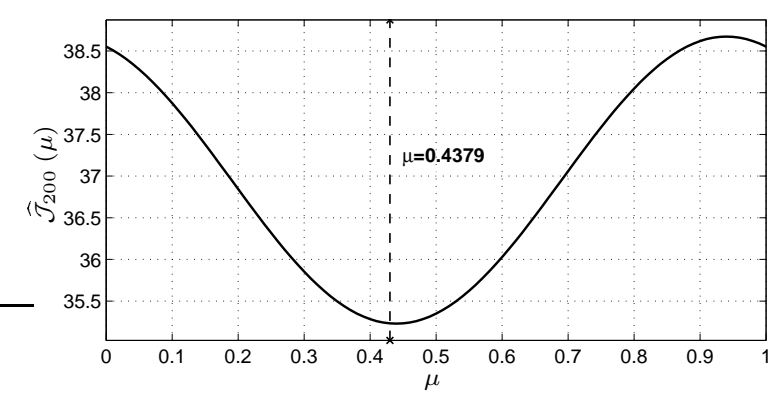

Fig. 2. Estimate of cost function $\widehat{\mathcal{J}}_{200}(\mu), K=200, \epsilon=0.43$ and $\mathrm{SNR}=15 \mathrm{~dB}$. The cost function is sinusoidal.

\section{PERFormance ANALYSis}

In this section, we assess the large sample performance of the proposed CFO estimator. First, both the finite sample covariance matrix and cost-function are given in closed-form. Then, we prove both the consistency of the estimator and the convergence in the mean square. Finally, we study the finite sample mean square error and conclude on the asymptotic behavior of the method. The convergence rate in mean square error is shown to be proportional to $1 / K$ as the sample size $K$ tends to infinity.

\section{A. Sample covariance matrix}

In practice, the covariance matrix $\widetilde{\mathbf{Q}}(\mu)$ has to be estimated at the receiver. Let $\widehat{\widetilde{\mathbf{Q}}}_{K}(\mu)$ be the sample estimate computed with a total number $K$ of observed blocks:

$$
\begin{aligned}
\widehat{\widetilde{\mathbf{Q}}}_{K}(\mu) & =\frac{1}{K} \sum_{k=0}^{K-1} \mathbf{v}_{\mu \epsilon}(k) \mathbf{v}_{\mu \epsilon}^{H}(k) \\
& =\mathbf{F}_{N}^{H} \mathbf{C}^{*}(\mu-\epsilon) \widehat{\mathbf{Q}}_{K} \mathbf{C}(\mu-\epsilon) \mathbf{F}_{N},
\end{aligned}
$$

where $\mathbf{v}_{\mu \epsilon}(k)$ denotes $\mathbf{v}_{\hat{\epsilon} \epsilon}(k)$ in (5) evaluated at $\hat{\epsilon}=\mu$, and $\widehat{\mathbf{Q}}_{K}$ denotes the sample covariance matrix in case of perfect frequency synchronization (i.e., no offset),

$$
\widehat{\mathbf{Q}}_{K}=\frac{1}{K} \sum_{k=0}^{K-1}[\widetilde{\mathbf{H}} \mathbf{b}(k)+\overline{\mathbf{w}}(k)][\widetilde{\mathbf{H}} \mathbf{b}(k)+\overline{\mathbf{w}}(k)]^{H},
$$

with $\overline{\mathbf{w}}(k)=\varphi_{k}^{*}(\epsilon) \mathbf{C}^{*}(\epsilon) \mathbf{w}(k)$. First, notice that the CFO does not change the noise statistics since the matrix $\varphi_{k}^{*}(\epsilon) \mathbf{C}^{*}(\epsilon)$ is unitary and the noise vector $\mathbf{w}(k)$ is assumed to be circular complex Gaussian. Second, the matrix $\widehat{\mathbf{Q}}_{K}$ can not be evaluated in practice because the received signal is corrupted by unknown frequency offset. However, it provides insight to the theoretical performance of the proposed algorithm as a function of the sample size.

\section{B. Consistency and convergence in the mean square}

In order to analyze the convergence of the algorithm as a function of the sample size, an alternate equivalent expression for the cost function is provided. With finite sample support of $K$ observations, the estimate of the cost function defined in (17) is expressed as

$$
\begin{aligned}
\widehat{\mathcal{J}}_{K}(\mu)=\| & \left(\mathbf{F}_{N}^{H} \mathbf{C}^{*}(\mu-\epsilon) \widehat{\mathbf{Q}}_{K} \mathbf{C}(\mu-\epsilon) \mathbf{F}_{N}\right) \odot \ldots \\
& \ldots \odot\left(\mathbb{1}_{N}-\mathbf{I}_{N}\right) \quad \|_{F}^{2}, \mu \in[0,1[.(26)
\end{aligned}
$$

Then, according to Lemma 1 with $\mathbf{R}=\widehat{\mathbf{Q}}_{K}$, and noticing that $\widehat{\mathcal{J}}_{K}(\mu)=\mathcal{K}_{\widehat{\mathbf{Q}}_{K}}(\mu-\epsilon)$, we get the following closedform expression

$$
\begin{aligned}
\widehat{\mathcal{J}}_{K}(\mu)= & a_{K}-\frac{2}{N} \operatorname{Re}\left\{\Gamma_{\widehat{\mathbf{Q}}_{K}}\right\} \cos (2 \pi(\mu-\epsilon)) \ldots \\
& \ldots-\frac{2}{N} \operatorname{Im}\left\{\Gamma_{\widehat{\mathbf{Q}}_{K}}\right\} \sin (2 \pi(\mu-\epsilon)),(27)
\end{aligned}
$$

where $a_{K} \in \mathbb{R}$, and $\Gamma_{\widehat{\mathbf{Q}}_{K}}$ is equal to $\Gamma_{\mathbf{R}}$ in the Proof of Lemma 1 (see Appendix II) evaluated at $\mathbf{R}=\widehat{\mathbf{Q}}_{K}$. The quantity $\Gamma_{\widehat{\mathbf{Q}}_{K}}$ represents the sample estimate of $\Gamma_{\mathbf{Q}}$ (see (31)), and is given by

$$
\Gamma_{\widehat{\mathbf{Q}}_{K}}=\sum_{\substack{u_{1}=0 \\\left(u_{1}-v_{1}\right)-\left(u_{2}-v_{2}\right)=N}}^{N-1} \sum_{u_{2}=0}^{N-1} \sum_{v_{1}=0}^{N-1} \hat{q}_{K, u_{1}, v_{1}} \hat{q}_{K, u_{2}, v_{2}}^{*} .
$$

The notation $\hat{q}_{K, u, v}$ refers to the $(u, v)$ element of the matrix $\widehat{\mathbf{Q}}_{K}$. Differentiating (27) with respect to $\mu$, the extrema of $\widehat{\mathcal{J}}_{K}(\mu)$ in $[0,1[$ are found as

$$
\hat{\mu}_{K_{i}}=\epsilon+\frac{1}{2 \pi} \arctan \frac{\operatorname{Im}\left\{\Gamma_{\widehat{\mathbf{Q}}_{K}}\right\}}{\operatorname{Re}\left\{\Gamma_{\widehat{\mathbf{Q}}_{K}}\right\}}+\frac{i}{2} \bmod 1, i=0,1 .
$$

Hence, the quantity $\Gamma_{\widehat{\mathbf{Q}}_{K}}$ and more specifically its phase plays a key role for the proposed blind CFO estimation algorithm, as it causes estimation errors. Notice that the distribution of $\Gamma_{\widehat{Q}_{K}}$ does not depend on $\epsilon$. Hence, the performance of the proposed estimator does not depend on the value of the CFO. The frequency offset estimate is found by choosing the value corresponding to the minimum of the cost function:

$$
\hat{\epsilon}_{K}= \begin{cases}\hat{\mu}_{K_{0}} & \text { if } \widehat{\mathcal{J}}_{K}\left(\hat{\mu}_{K_{0}}\right)<\widehat{\mathcal{J}}_{K}\left(\hat{\mu}_{K_{1}}\right) \\ \hat{\mu}_{K_{1}} & \text { otherwise. }\end{cases}
$$

\footnotetext{
${ }^{4}$ Extrema in (29) are indeed identical to those of (21), except that their respective ordering may differ.
} 
Now, Lemma 2 below plays a central role in establishing the asymptotic performance of the algorithm.

Lemma 2: Let

$$
\Gamma_{\mathbf{Q}}=\sum_{\substack{u_{1}=0 \\\left(u_{1}-v_{1}\right)}}^{N-1} \sum_{u_{2}=0}^{N-1} \sum_{\left.u_{2}-v_{2}\right)=0}^{N-1} \sum_{v_{2}=0}^{N-1} q_{u_{1}, v_{1}} q_{u_{2}, v_{2}}^{*},
$$

with $q_{u, v}$ defined as a general element of $\mathbf{Q}$ in (11). Moreover, let $\Gamma_{\widehat{\mathbf{Q}}_{K}}$ be as given in (28). Then,

1) As $K \rightarrow \infty, \Gamma_{\widehat{\mathbf{Q}}_{K}} \rightarrow \Gamma_{\mathbf{Q}}$ w.p. 1 .

2) $\operatorname{Im}\left\{\Gamma_{\mathbf{Q}}\right\}=0$ and $\operatorname{Re}\left\{\Gamma_{\mathbf{Q}}\right\}>0$.

Proof is given in Appendix III. As a consequence of the above lemma and equations (29)-(30), we get the following:

Theorem 2 (Strong consistency): As $K \rightarrow \infty$,

$$
\hat{\epsilon}_{K} \rightarrow \epsilon \text { w.p. } 1 \text {. }
$$

Proof is given in Appendix IV. As convergence with probability 1 implies convergence in probability [35, p.10], the proposed estimator yields a consistent estimate of the CFO $\epsilon$. In addition, convergence in the mean square follows from Theorem 2 and from the fact that $\hat{\epsilon}_{K}$ is bounded:

Corollary 1: $\hat{\epsilon}_{K}$ converges to $\epsilon$ in the mean square.

Proof is given in Appendix V. Consequently, the proposed estimator is asymptotically unbiased.

\section{Convergence rate}

Corollary 1 showed that the mean square error (MSE) of $\hat{\epsilon}_{K}$ tends to zero as $K \rightarrow \infty$. We next study the convergence rate of the estimator. Let us define the MSE after $K$ received blocks by

$$
\operatorname{MSE}_{K}=\mathrm{E}\left[\left(\epsilon-\hat{\epsilon}_{K}\right)^{2}\right]
$$

From expression (29), and by using similar arguments to [36]-[38], we may approximate the MSE as

$$
\operatorname{MSE}_{K} \cong \frac{1}{4 \pi^{2}} \frac{\mathrm{E}\left[\operatorname{Im}^{2}\left\{\Gamma_{\widehat{\mathbf{Q}}_{K}}\right\}\right]}{\mathrm{E}\left[\operatorname{Re}^{2}\left\{\Gamma_{\widehat{\mathbf{Q}}_{K}}\right\}\right]} .
$$

It is shown in Appendix VI that the expression in (33) may be written as a quotient of polynomials. Hence the approximated MSE becomes

$$
\operatorname{MSE}_{K} \cong \frac{1}{4 \pi^{2}} \frac{\mathcal{P}^{(\leq 3)}(K)}{\mathcal{Q}^{(4)}(K)} \propto 1 / K,
$$

where $\mathcal{Q}^{(4)}$ is a polynomial of degree four in $K$, while the polynomial $\mathcal{P}^{(\leq 3)}$ is of degree at most three. Hence, it follows that for $K$ sufficiently large, the rate of convergence in MSE is proportional to $1 / K$.

\section{CRAMÉR-RAO BOUND}

To assess the large sample performance of the proposed method, we derive the Cramér-Rao bound (CRB), under the assumption that the transmitted symbol vector $\mathbf{b}$ in (1) is Gaussian. Since we are interested in both real and complex modulations, $\mathbf{b}$ cannot always be modeled as a complex circular random vector. Complete second order statistics require both signal covariance and pseudocovariance matrices (see Section III and [33]).

The Gaussian approximation has been widely used in the literature related to OFDM [11], [20]. This approximation is justified by the central limit theorem. Hence, we may assume that the vector $\mathbf{s}(k)=\widetilde{\mathbf{H}} \mathbf{F}_{N} \mathbf{a}(k)$ is multivariate Gaussian. Then, the OFDM transmission with imperfect frequency synchronization in (2) may be written using the model

$$
\mathbf{z}(k)=\overline{\mathbf{C}}_{\epsilon, k} \mathbf{s}(k)+\mathbf{w}(k),
$$

where $\overline{\mathbf{C}}_{\epsilon, k}=\varphi_{k}(\epsilon) \mathbf{C}_{\epsilon}$ and $\mathbf{w}$ is the complex circular Gaussian noise vector. The stochastic CRB for blind frequency offset estimation in OFDM and the associated Fisher information matrix (FIM) with finite sample size $K$ are respectively given by

$$
\begin{aligned}
\mathrm{CRB}_{K}(\epsilon) & =\left[\mathrm{FIM}_{K}^{-1}\right]_{1,1} \\
\mathrm{FIM}_{K} & =\frac{1}{2} \sum_{k=0}^{K-1}\left[\begin{array}{c}
\mathbf{g}_{k}^{H} \\
\boldsymbol{\Delta}_{k}^{H}
\end{array}\right]\left[\begin{array}{ll}
\mathbf{g}_{k} & \boldsymbol{\Delta}_{k}
\end{array}\right],
\end{aligned}
$$

where the vector $\mathbf{g}_{k} \in \mathbb{C}^{4 N^{2} \times 1}$ and the matrix $\boldsymbol{\Delta}_{k} \in$ $\mathbb{C}^{4 N^{2} \times(M+1)}$ are defined respectively as

$$
\begin{aligned}
\mathbf{g}_{k} & =\operatorname{vec}\left(\boldsymbol{\Omega}_{k}^{-1 / 2}\left(\widetilde{\mathbf{D}}_{\epsilon, k} \widetilde{\boldsymbol{\Omega}} \widetilde{\mathbf{C}}_{\epsilon, k}^{H}+\widetilde{\mathbf{C}}_{\epsilon, k} \widetilde{\boldsymbol{\Omega}} \widetilde{\mathbf{D}}_{\epsilon, k}^{H}\right) \boldsymbol{\Omega}_{k}^{-1 / 2}\right) \\
\boldsymbol{\Delta}_{k} & =\left[\begin{array}{ll}
\mathbf{V}_{k} & \mathbf{u}_{k}
\end{array}\right], \mathbf{V}_{k} \in \mathbb{C}^{4 N^{2} \times M}, \mathbf{u}_{k} \in \mathbb{C}^{4 N^{2} \times 1},
\end{aligned}
$$

with the following notation:

- Covariance matrix of $\mathbf{z}(k): \boldsymbol{\Omega}_{k}=\widetilde{\mathbf{C}}_{\epsilon, k} \widetilde{\boldsymbol{\Omega}} \widetilde{\mathbf{C}}_{\epsilon, k}^{H}$.

- Inverse of $\boldsymbol{\Omega}_{k}$ in vector form: $\mathbf{u}_{k}=\operatorname{vec}\left(\boldsymbol{\Omega}_{k}^{-1}\right)$.

- Covariance matrix of s: $\widetilde{\boldsymbol{\Omega}}=\left[\begin{array}{ll}\mathbf{Q} & \mathbf{P} \\ \mathbf{P}^{H} & \mathbf{Q}^{*}\end{array}\right]$.

- $\mathbf{Q}=\operatorname{cov}(\mathbf{r})$ and $\mathbf{P}=\operatorname{pcov}(\mathbf{r})$ where $\mathbf{r}$ is as in (4).

- Frequency offset matrix and its derivative wrt. to $\epsilon$ :

$$
\begin{gathered}
\widetilde{\mathbf{C}}_{\epsilon, k}=\left[\begin{array}{ll}
\overline{\mathbf{C}}_{\epsilon, k} & \mathbf{0} \\
\mathbf{0} & \overline{\mathbf{C}}_{\epsilon, k}^{*}
\end{array}\right], \widetilde{\mathbf{D}}_{\epsilon, k}=\frac{\partial}{\partial \epsilon} \widetilde{\mathbf{C}}_{\epsilon, k} . \\
\text { - } \mathbf{V}_{k}=\left(\left(\boldsymbol{\Omega}_{k}^{-1 / 2} \widetilde{\mathbf{C}}_{\epsilon, k}^{*}\right) \otimes\left(\boldsymbol{\Omega}_{k}^{-1 / 2} \widetilde{\mathbf{C}}_{\epsilon, k}\right)\right) \mathbf{J} .
\end{gathered}
$$

The matrix $\mathbf{J}$ above is such that $\operatorname{vec}(\widetilde{\boldsymbol{\Omega}})=\mathbf{J} \boldsymbol{\rho}$, and the entire statistics $\{\mathbf{P}, \mathbf{Q}\}$ depend only on a finite number $M$ of real-valued unknown parameters stacked into the vector $\boldsymbol{\rho}=\left[\rho_{1}, \ldots, \rho_{M}\right]^{T}$. Detailed derivations may be found in [38] and [39]. Note that an alternate expression for the stochastic CRB for a similar model has also been independently derived in [24]. Even though the component-wise Gaussian approximation is reasonable, the assumption on the joint Gaussianity of the compo- 
nents is more questionable, and this may lead to slight differences compared to the exact CRB.

\section{Simulations}

In this section, simulation results are reported. Performance is compared to the Cramér-Rao bound derived in Section VI. Asymptotic results presented in Section V are validated through numerical experiments as well.

\section{A. Simulation parameters}

1) OFDM parameters: The OFDM system parameters are chosen as follows: the carrier frequency is $f_{0}=2.4$ $\mathrm{GHz}$, the number of subcarriers is set to $N=64$ and the available bandwidth is $B=0.5 \mathrm{MHz}$. The length $L$ of the cyclic prefix is 4 . The subcarrier symbol rate is of $7.8 \mathrm{KHz}$. QPSK and 16QAM symbol modulations are considered. The normalized frequency offset is $\epsilon=$ 0.43. In most of the simulations, independent runs of the algorithm over $K$ OFDM blocks are performed. This is referred to as a realization. Ensemble averages of the quantities of interest are computed over 100 realizations.

2) Channel model: We proceed in two steps. First, to allow the comparison with the CRB, the wireless channel is considered to be deterministic but unknown to the receiver. The channel impulse response chosen for our simulations has four transmission paths and is the following:

$$
\mathbf{h}=\left[\begin{array}{r}
0.0731-0.8702 j \\
0.3613-0.4503 j \\
-0.1098+0.4476 j \\
-0.0270-0.0942 j
\end{array}\right]
$$

As a second step, a 4-tap random channel impulse response is chosen for each realization. Channel coefficients are assumed to be i.i.d. Rayleigh distributed with unit variance.

\section{B. Performance of the proposed algorithm}

First, convergence as a function of sample size is investigated. Blind frequency offset estimation over one realization and the associated value of the cost function are shown in Figure 3. The sample estimate of received signal covariance matrix improves over time as more OFDM blocks become available. Consequently, the estimate of the CFO gets more accurate as well. The speed of convergence may be significantly improved by enforcing a Toeplitz structure for the sample covariance matrix in time domain, i.e., by averaging over its diagonals.

The reduction of the level of inter-carrier interference is depicted in Figure 4 where the covariance matrix of the signal in frequency domain is plotted before and after compensation for the frequency offset. Dark colors in the figure correspond to high absolute values. As shown by Figure 4, accurate offset compensation efficiently removes off-diagonal terms caused by ICI and restores the initial
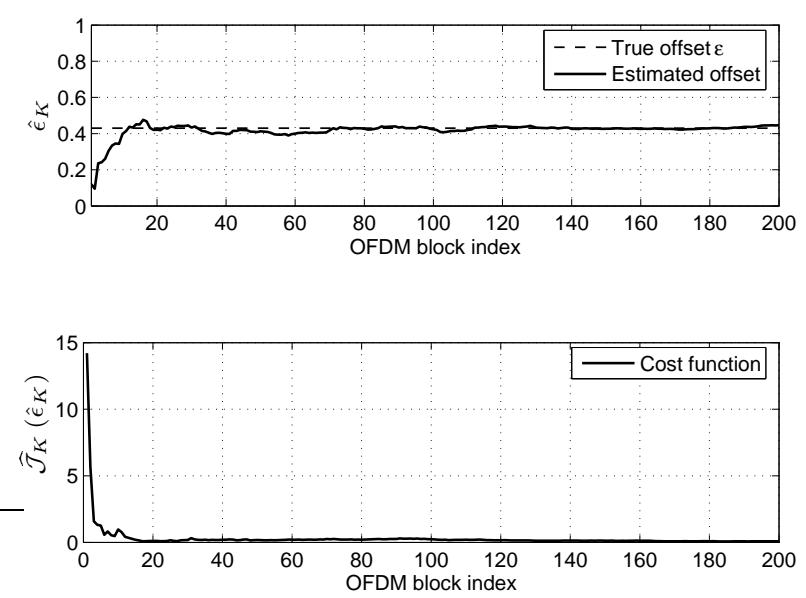

Fig. 3. Estimated frequency offset and cost function over time (1 realization), $\epsilon=0.43$ and $\mathrm{SNR}=15 \mathrm{~dB}$.
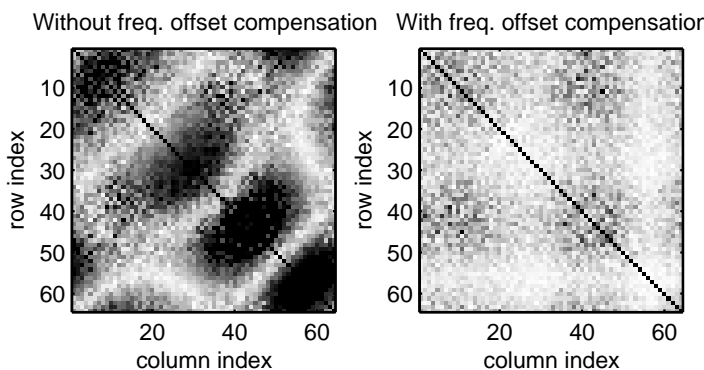

Fig. 4. Covariance matrix structure without (on the left) and with (on the right) frequency offset compensation for $\epsilon=0.43$, QPSK modulation and $\mathrm{SNR}=15 \mathrm{~dB}$. Dark colors correspond to high absolute values. Estimation has been performed over 10000 blocks. Total off-diagonal power gets reduced by $19.8 \mathrm{~dB}$. In successful CFO compensation, nondiagonal elements are zero and only the diagonal terms remain.

orthogonality of the OFDM transmission. The initial offdiagonal power is reduced by more than $19 \mathrm{~dB}$, after $K=$ 10000 blocks.

Then, we present results in terms of mean square error (see eq. (32)), which is chosen as error criterion for offset estimation. The plot of the MSE versus the number of observed blocks at $10 \mathrm{~dB}$ SNR is depicted in Figure 5. The MSE falls below $5.10^{-4}$ ( $5 \%$ residual error on the CFO) after 100 blocks on average. The MSE reaches $7.10^{-5}$ after 600 blocks, leaving a residual error less than $2 \%$ for both QPSK and 16QAM modulations. The difference in performance between fixed and randomly chosen channel impulse responses is not significant. OFDM systems are commonly considered to tolerate synchronization errors up to a few percents of the carrier spacing [2]. For instance, QPSK modulation may tolerate up to $5 \%$ error whereas 64QAM requires at least $1 \%$ accuracy for a loss of $0.5 \mathrm{~dB}$ in SNR [40]. Hence, the proposed approach is a feasible solution for practical receivers.

The graph of the MSE versus SNR is depicted in Figure 
6 for sample sizes $K=200,2000$ blocks. As expected, there is no significant dependence of the performance on the noise level. In theory, the covariance matrix of the noise is diagonal on average for complex white noise. Hence, the performance of the algorithm should not depend on the SNR if noise is uncorrelated. However, in practice the sample estimates of the covariance matrix experience perturbations because of noise which degrades the performance at small sample size and low SNR.

The mean square error of the estimator is plotted against the stochastic CRB derived in Section VI (Fig. 5 and 6). The proposed method may be biased for small sample size (gap to the CRB in Figure 6). But, there is no error floor present in the plot of the MSE as a function of the sample size (Fig. 5). Indeed, the MSE curve stays parallel to the CRB. These remarks support the claim of asymptotic unbiasedness. However, even though the CRB is not attained, the residual estimation error is small (less than $2 \%$ on average after 600 blocks). Hence, the proposed method performs accurately almost regardless of the SNR.

Unlike [25], the proposed method performs well for multipath channels with unknown channel length, provided that the latter is smaller than the duration of the cyclic prefix. In addition, as seen in Figure 6, the algorithm performs equally well for QPSK and 16QAM modulations ${ }^{5}$ and offers robust performance at low SNR. Hence, the method allows efficient use of the spectrum as it is not restricted to constant modulus constellations. Note that no pilot or virtual subcarriers are exploited here. VC usually improve the performance of blind CFO estimation significantly [16], [23], as they induce a low rank signal model, which allows using high resolution subspace techniques. But this is at the expense of bandwidth efficiency, since virtual carriers do not carry any information symbols.

\section{Convergence rate}

It was shown in Subsection V-C that for large sample size $K$, the MSE decreases as $1 / K$. In Figure 7 , the quantity $K \times \mathrm{MSE}_{K}$ is plotted as a function of the sample size. Curves are ensemble averages over 100 realizations. It is clearly seen from Figure 7 that the product $K \times \mathrm{MSE}_{K}$ is approximately constant for $K \geq 200$ blocks, for both fixed and randomly chosen channels impulse responses. Hence numerical results validate the previously derived asymptotic result. Furthermore, theoretical analysis conducted in Section $\mathrm{V}$ did not show any dependence on the value of the CFO of the distribution of the error term. Hence, the performance of the proposed estimator does not depend on the value of the CFO $\epsilon$ in the interval $[0,1[$.

\footnotetext{
${ }^{5}$ Identical performance is achieved with complex random Gaussian distributed signals as well.
}

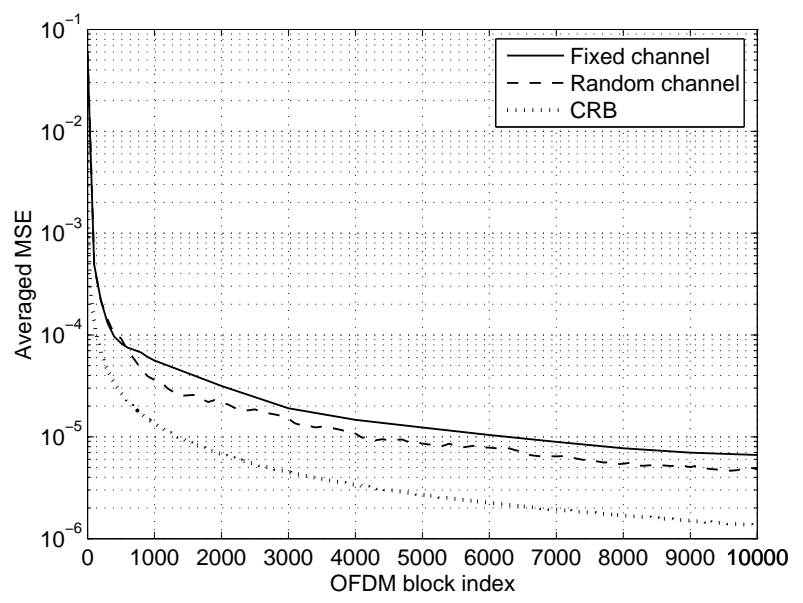

Fig. 5. MSE and stochastic CRB vs. number of observed blocks for fixed and random channel impulse responses at $\mathrm{SNR}=10 \mathrm{~dB}$; ensemble average over 100 realizations; $\epsilon=0.43$; QPSK modulation. No error floor as the sample size increases, since convergence rate in MSE is proportional to $1 / K$.

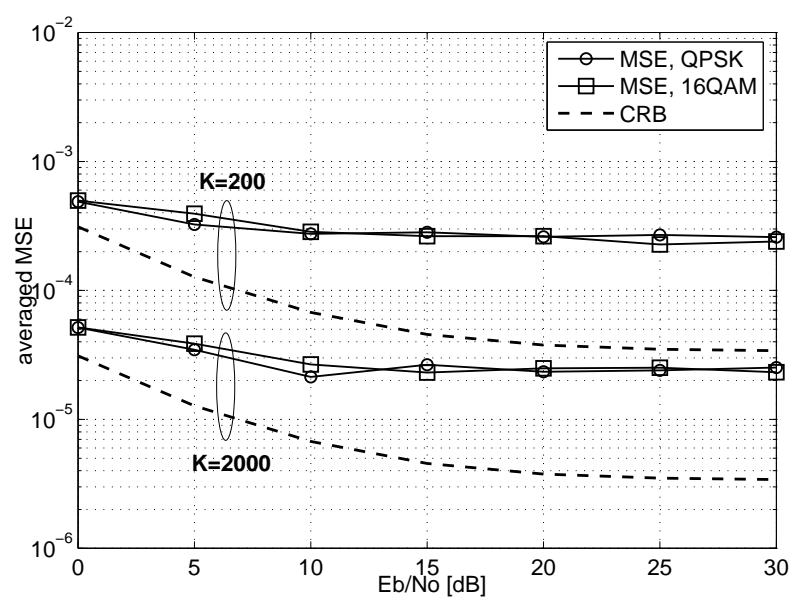

Fig. 6. MSE and stochastic CRB vs. SNR for QPSK and 16QAM modulations ${ }^{5}$; fixed channel; $K=200$ and $K=2000$ blocks; ensemble average over 100 realizations; $\epsilon=0.43$. With small sample sizes bias may be present (error floor), but estimator is asymptotically unbiased. Residual error remains well below $2 \%$.

\section{CONCLUSIONS}

In this paper, we introduced a blind frequency offset estimator for OFDM systems using real- and complexvalued modulations, under frequency selective fading. It is based on the property that perfect carrier frequency synchronization implies a diagonal covariance matrix for the received signal in frequency domain. Cost function minimization is accomplished in a closed-form, which leads to an accurate and computationally efficient solution. No knowledge of the underlying multipath wireless channel is required, nor the use of virtual carriers. Channel estimation may then be performed as a subsequent step, after the frequency synchronization is achieved. The 


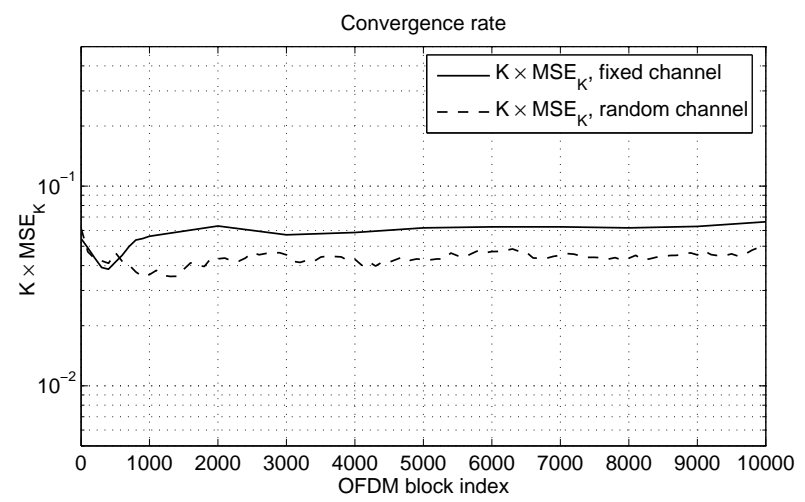

Fig. 7. Analysis of the convergence rate: plot of $K \times \mathrm{MSE}_{K}$ over time, for fixed and random channels; $\mathrm{SNR}=5 \mathrm{~dB}$; ensemble average over 100 realizations. The product $K \times \mathrm{MSE}_{K}$ is approximately constant for $K \geq 200$ blocks. Hence, the MSE decreases as $1 / K$ as $K$ tends to infinity. Convergence of the MSE as a function of the sample size is depicted in Figure 5.

performance remains practically constant regardless of the SNR if the noise is white. The proposed method is shown to yield a consistent estimate of the CFO. Hence, it is asymptotically unbiased. The proposed CFO estimator performs close to the (stochastic) Cramér-Rao bound established for the general multivariate Gaussian model.

\section{APPENDIX I}

\section{PROOF OF THEOREM 1}

Proof: 1) For $\mu=\epsilon, \mathbf{C}(0)=\mathbf{I}_{N}$ and the matrix $\widetilde{\mathbf{Q}}(\mu)$ in (12) becomes

$$
\widetilde{\mathbf{Q}}(\epsilon)=\mathbf{F}_{N}^{H} \widetilde{\mathbf{H}} \widetilde{\mathbf{H}}^{H} \mathbf{F}_{N}+\sigma^{2} \mathbf{I}_{N} .
$$

Now, the matrix $\mathbf{F}_{N}^{H} \widetilde{\mathbf{H}} \widetilde{\mathbf{H}}^{H} \mathbf{F}_{N}$ in (38) is diagonal as $\widetilde{\mathbf{H}} \widetilde{\mathbf{H}}^{H}$ is circulant as a product of two circulant matrices. Thus, since $\sigma^{2} \mathbf{I}_{N}$ is diagonal, $\widetilde{\mathbf{Q}}(\epsilon)$ is diagonal as well and $\mathcal{J}(\epsilon)=0$.

2) A straightforward way to prove uniqueness of the minimum is to apply Lemma 1 with $\mathbf{R}=\mathbf{Q}$ in (11), and to notice that $\mathcal{J}(\mu)=\mathcal{K}_{\mathbf{Q}}(\mu-\epsilon)$. Then,

$$
\begin{array}{r}
\mathcal{J}(\mu)=a-\frac{2}{N} \operatorname{Re}\left\{\Gamma_{\mathbf{Q}}\right\} \cos (2 \pi(\mu-\epsilon)) \ldots \\
\ldots-\frac{2}{N} \operatorname{Im}\left\{\Gamma_{\mathbf{Q}}\right\} \sin (2 \pi(\mu-\epsilon)),
\end{array}
$$

where $a \in \mathbb{R}$ and $\Gamma_{\mathbf{Q}}$ are given as in the proof of Lemma 1. As a consequence of Lemma $2, \operatorname{Re}\left\{\Gamma_{\mathbf{Q}}\right\}>0$, and $\operatorname{Im}\left\{\Gamma_{\mathbf{Q}}\right\}=0$. Then, the cost function may be rewritten as

$$
\mathcal{J}(\mu)=a-\frac{2}{N} \operatorname{Re}\left\{\Gamma_{\mathbf{Q}}\right\} \cos (2 \pi(\mu-\epsilon)) .
$$

From (40) and since $\operatorname{Re}\left\{\Gamma_{\mathbf{Q}}\right\}>0$, the function $\mathcal{J}(\mu)$ is obviously periodic with period 1 and has unique minimum at $\epsilon$ in $[0,1[$. Hence, $\mathcal{J}(\mu)>\mathcal{J}(\epsilon), \forall \mu \neq \epsilon, \mu \in[0,1[$.

\section{APPENDIX II \\ PROOF OF LEMMA 1}

Proof: First, let us define the matrix $\mathbf{M}(\mu)$ of size $N \times N$ as $\mathbf{M}(\mu)=\mathbf{F}_{N}^{H} \mathbf{C}^{*}(\mu) \mathbf{R C}(\mu) \mathbf{F}_{N}$, with its $(s, t)$ element given by

$$
m_{s, t}=\frac{1}{N} \sum_{u_{1}=0}^{N-1} \sum_{v_{1}=0}^{N-1} e^{-\frac{2 \pi j\left(u_{1} s-v_{1} t+\left(u_{1}-v_{1}\right) \mu\right)}{N}} r_{u_{1}, v_{1}},
$$

with $s, t=0, \ldots, N-1$ and where $r_{u_{1}, v_{1}}$ is the $\left(u_{1}, v_{1}\right)$ element of the matrix $\mathbf{R}$ of size $N \times N$. Then, $\mathcal{K}_{\mathbf{R}}$ is expressed as

$$
\mathcal{K}_{\mathbf{R}}(\mu)=\left\|\mathbf{M}(\mu) \odot\left(\mathbb{1}_{N}-\mathbf{I}_{N}\right)\right\|_{F}^{2}=\sum_{s=0}^{N-1} \sum_{\substack{t=0 \\ t \neq s}}^{N-1}\left|m_{s, t}\right|^{2} .
$$

Inserting $\left|m_{s, t}\right|^{2}=m_{s, t} m_{s, t}^{*}$ from (41) into (42) and rearranging terms leads to

$$
\begin{gathered}
\mathcal{K}_{\mathbf{R}}(\mu)=\frac{1}{N^{2}} \sum_{u_{1}=0}^{N-1} \sum_{u_{2}=0}^{N-1} \sum_{v_{1}=0}^{N-1} \sum_{v_{2}=0}^{N-1} e^{-\frac{2 \pi j\left[\left(u_{1}-u_{2}\right)-\left(v_{1}-v_{2}\right)\right] \mu}{N}} \ldots \\
\ldots r_{u_{1}, v_{1}} r_{u_{2}, v_{2}}^{*} \sum_{s=0}^{N-1} e^{-\frac{2 \pi j\left(u_{1}-u_{2}\right) s}{N}} \sum_{\substack{t=0 \\
t \neq s}}^{N-1} e^{\frac{2 \pi j\left(v_{1}-v_{2}\right) t}{N}} .
\end{gathered}
$$

First, we notice that

$$
\sum_{\substack{t=0 \\ t \neq s}}^{N-1} e^{\frac{2 \pi j\left(v_{1}-v_{2}\right) t}{N}}=\sum_{t=0}^{N-1} e^{\frac{2 \pi j\left(v_{1}-v_{2}\right) t}{N}}-e^{\frac{2 \pi j\left(v_{1}-v_{2}\right) s}{N}} .
$$

Second, we recall the following well known result:

$$
\forall v \in \mathbb{Z}, \sum_{u=0}^{N-1} e^{j \frac{2 \pi u v}{N}}=\left\{\begin{array}{l}
N, \text { if } v=k N, k \in \mathbb{Z} . \\
0, \text { otherwise. }
\end{array}\right.
$$

Finally, by applying (44) and (45) onto (43) we get the following closed-form expression for $\mathcal{K}_{\mathbf{R}}(\mu)$ :

$$
\begin{gathered}
\mathcal{K}_{\mathbf{R}}(\mu)=a+b \cos (2 \pi \mu)+c \sin (2 \pi \mu) \\
a=\sum_{u_{1}=0}^{N-1} \sum_{v_{1}=0}^{N-1}\left|r_{u_{1}, v_{1}}\right|^{2} \ldots \\
\ldots-\frac{1}{N} \sum_{u_{1}=0}^{N-1} \sum_{u_{2}=0}^{N-1} \sum_{v_{1}=0}^{N-1} \sum_{v_{2}=0}^{N-1} r_{u_{1}, v_{1}} r_{u_{2}, v_{2}}^{*} \\
b=-\frac{2}{N} \operatorname{Re}\left\{\Gamma_{\mathbf{R}}\right\}, \quad c=-\frac{2}{N} \operatorname{Im}\left\{\Gamma_{\mathbf{R}}\right\} \\
\Gamma_{\mathbf{R}}=\sum_{\substack{\left(u_{1}-v_{1}\right)-\left(u_{2}-v_{2}\right)=0 \\
u_{1}=0}}^{N-1} \sum_{u_{2}=0}^{N-1} \sum_{v_{1}=0}^{N-1} \sum_{v_{2}=0}^{N-1} r_{u_{1}, v_{1}} r_{u_{2}, v_{2}}^{*} \\
\left.u_{u_{2}}-v_{2}\right)=N
\end{gathered}
$$




\section{APPENDIX III \\ PROOF OF LEMMA 2}

Proof: We start by proving the part 1). The strong law of large numbers (see, for example, Theorem $1.8 \mathrm{D}$ in [35]) implies that $\widehat{\mathbf{Q}}_{K} \rightarrow \mathbf{Q}$ w.p. 1 , as $K \rightarrow \infty$. Using this result and Theorem 1.7 in [35, p.24], we get that

$$
\hat{q}_{K, u_{1}, v_{1}} \hat{q}_{K, u_{2}, v_{2}}^{*} \rightarrow q_{u_{1}, v_{1}} q_{u_{2}, v_{2}}^{*} \quad \text { w.p. } 1,
$$

as $K \rightarrow \infty$, for any $u_{1}, v_{1}, u_{2}, v_{2}=0, \ldots, N-1$. Therefore, also

$$
\begin{aligned}
& \sum_{\begin{array}{c}
u_{1}=0 \\
\left(u_{1}-v_{1}\right)
\end{array}}^{N-1} \sum_{u_{2}=0}^{N-1} \sum_{v_{1}=0}^{N-1} \sum_{u_{2}=0}^{N-1} \hat{q}_{K, u_{1}, v_{1}} \hat{q}_{K, u_{2}, v_{2}}^{*} v_{2} \\
& \rightarrow \sum_{\begin{array}{c}
u_{1}=0 \\
\left(u_{1}-v_{1}\right)-\left(u_{2}-v_{2}\right)=N
\end{array}}^{N-1} \sum_{u_{2}=0}^{N-1} \sum_{v_{1}=0}^{N-1} \sum_{v_{2}=0}^{N-1} q_{u_{1}, v_{1}} q_{u_{2}, v_{2}}^{*} \text { w.p. } 1
\end{aligned}
$$

as $K \rightarrow \infty$, which completes the proof of part 1 ).

We now continue by proving the part 2). The proof is based on structural properties of circulant matrices. It can be verified that matrix $\mathbf{Q}=\widetilde{\mathbf{H}} \widetilde{\mathbf{H}}^{H}+\sigma^{2} \mathbf{I}_{N}$ in (11) is circulant $\left(\widetilde{\mathbf{H}} \widetilde{\mathbf{H}}^{H}\right.$ is circulant as $\widetilde{\mathbf{H}}$ is a circulant matrix, and $\sigma^{2} \mathbf{I}_{N}$ is a diagonal matrix). As $\mathbf{Q}$ is circulant, it first inherits the Toeplitz structure (equal elements along the diagonals). Hence, $q_{u_{1}, v_{1}}$ in (31) may be replaced by $q_{s}$, with $s=u_{1}-v_{1}, s=-(N-1), \ldots, N-1$. Based on this observation, we rewrite the sum $\Gamma_{\mathbf{Q}}$ as

$$
\Gamma_{\mathbf{Q}}=\sum_{s=-(N-1)}^{N-1} \sum_{\substack{t=-(N-1) \\ s-t=N}}^{N-1} \beta_{s} \beta_{t} q_{s} q_{t}^{*}
$$

where we made the change in variables $s=u_{1}-v_{1}$ and $t=u_{2}-v_{2}$. Notice that indices $s$ and $t$ both index diagonals (i.e. as the difference of row and column indices). The number of elements on the diagonals indexed by $s$ and $t$ are denoted $\beta_{s}$ and $\beta_{t}$, respectively. Now, the constraint $s-t=N$ with $s, t=-(N-1), \ldots, N-1$ translates into $t=s-N$ with $s=1, \ldots, N-1$. Then, equation (52) becomes

$$
\Gamma_{\mathbf{Q}}=\sum_{s=1}^{N-1} \beta_{s} \beta_{s-N} q_{s} q_{s-N}^{*}=\sum_{s=1}^{N-1} \beta_{s} \beta_{s-N}\left|q_{s}\right|^{2},
$$

where the second equality is due to the fact that $\mathbf{Q}$ is circulant, i.e., $q_{u_{1}, v_{1}}=q_{\left(u_{1}-v_{1}\right)} \bmod N$. Thus, $q_{s}=q_{s-N}$.

Since $\beta_{s}>0, \beta_{s-N}>0$ and $\left|q_{s}\right|^{2} \geq 0$, we hence conclude that $\Gamma_{\mathbf{Q}} \geq 0$ and $\Gamma_{\mathbf{Q}} \in \mathbb{R}$. Moreover, assuming a non-diagonal channel covariance matrix $\widetilde{\mathbf{H}} \widetilde{\mathbf{H}}^{H}$, there exists at least one non-zero off-diagonal term in $\mathbf{Q}=$ $\widetilde{\mathbf{H}} \widetilde{\mathbf{H}}^{H}+\sigma^{2} \mathbf{I}_{N}$, and thus $\Gamma_{\mathbf{Q}}>0$.

\section{APPENDIX IV}

\section{PROOF OF THEOREM 2}

Proof: First, as a consequence of Lemma 2, as $K \rightarrow \infty, \operatorname{Re}\left\{\Gamma_{\widehat{\mathbf{Q}}_{K}}\right\} \rightarrow \operatorname{Re}\left\{\Gamma_{\mathbf{Q}}\right\}$ w.p. 1 and $\operatorname{Im}\left\{\Gamma_{\widehat{\mathbf{Q}}_{K}}\right\} \rightarrow$
0 w.p. 1. Using Theorem 1.7 in [35, p.24] and the fact that $\operatorname{Re}\left\{\Gamma_{\mathbf{Q}_{K}}\right\}>0$ (see Lemma 2), we then get that $\frac{\operatorname{Im}\left\{\Gamma_{\widehat{\mathbf{Q}}_{K}}\right\}}{\operatorname{Re}\left\{\Gamma_{\widehat{\mathbf{Q}}_{K}}\right\}} \rightarrow 0$ w.p. 1 as $K \rightarrow \infty$. Now, since arctan is differentiable in the neighborhood of 0 , we finally obtain that $\arctan \frac{\operatorname{Im}\left\{\Gamma_{\widehat{\mathbf{Q}}_{K}}\right\}}{\operatorname{Re}\left\{\Gamma_{\widehat{\mathrm{Q}}_{K}}\right\}} \rightarrow 0$ w.p. 1 as $K \rightarrow \infty$ (see Theorem 1.7 in [35, p.24]). Hence, by using the form in (29), we obtain the convergence of the extrema, as $K \rightarrow \infty$,

$$
\hat{\mu}_{K_{i}} \rightarrow \epsilon+\frac{i}{2} \quad \bmod 1 \quad \text { w.p. } 1, \quad i=0,1 .
$$

Second, by applying Lemma 2, it follows from (27) that

$$
\widehat{\mathcal{J}}_{K}(\mu) \rightarrow \mathcal{J}(\mu) \text { w.p. } 1, \forall \mu \in[0,1[,
$$

where $\mathcal{J}(\mu)$ is defined as in (40). Therefore, as $K \rightarrow \infty$, $\widehat{\mathcal{J}}_{K}\left(\hat{\mu}_{K_{0}}\right)<\widehat{\mathcal{J}}_{K}\left(\hat{\mu}_{K_{1}}\right)$ w.p. 1, and we get that $\hat{\epsilon}_{K} \rightarrow$ $\hat{\mu}_{K_{0}}$ w.p. 1 , as $K \rightarrow \infty$. Finally (54) implies that $\hat{\mu}_{K_{0}} \rightarrow$ $\epsilon$ w.p. 1 , as $K \rightarrow \infty$.

\section{APPENDIX V \\ PROOF OF COROLlaRY 1}

Proof: Dominated convergence w.p. 1 implies convergence in $r$-th mean (cf. [35], page 11). Hence, since $\hat{\epsilon}_{K} \rightarrow \epsilon$ w.p. 1 as $K \rightarrow \infty$, and $\left|\hat{\epsilon}_{K}\right| \leq 1$, then $\hat{\epsilon}_{K}$ converges to $\epsilon$ in the mean square $(r=2)$.

\section{APPENDIX VI \\ CONVERGENCE RATE}

Proof: We provide here a sketch of the derivation for the finite sample MSE as well as its rate of convergence, due to lack of space. Detailed derivation follow the ones in [39]. Let us start from the approximated MSE (see eq. (33) in Section V-C). We derive expressions for the quantities $\mathrm{E}\left[\operatorname{Im}^{2}\left\{\Gamma_{\widehat{\mathbf{Q}}_{K}}\right\}\right]$ and $\mathrm{E}\left[\operatorname{Re}^{2}\left\{\Gamma_{\widehat{\mathbf{Q}}_{K}}\right\}\right]$ in terms of polynomials in the sample size $K$, as follows:

$$
\begin{aligned}
\mathrm{E}\left[\operatorname{Im}^{2}\left\{\Gamma_{\widehat{\mathbf{Q}}_{K}}\right\}\right] & =\frac{1}{K^{4}}\left\{K^{4} \operatorname{Im}\left\{\Gamma_{\mathbf{Q}}\right\}^{2}+\mathcal{P}^{(\leq 3)}(K)\right\} \\
& =\frac{1}{K^{4}} \mathcal{P}^{(\leq 3)}(K) \\
\mathrm{E}\left[\operatorname{Re}^{2}\left\{\Gamma_{\widehat{\mathbf{Q}}_{K}}\right\}\right] & =\frac{1}{K^{4}}\left\{K^{4} \operatorname{Re}\left\{\Gamma_{\mathbf{Q}}\right\}^{2}+\mathcal{Q}^{(\leq 3)}(K)\right\},
\end{aligned}
$$

where $\mathcal{P}^{(\leq 3)}$ and $\mathcal{Q}^{(\leq 3)}$ are polynomials of degree at most three in $K$, and $\operatorname{Re}\left\{\Gamma_{\mathbf{Q}}\right\}>0, \operatorname{Im}\left\{\Gamma_{\mathbf{Q}}\right\}=0$ follows from Lemma 2. The term $\mathrm{E}\left[\operatorname{Re}^{2}\left\{\Gamma_{\widehat{\mathbf{Q}}_{K}}\right\}\right]$ is obviously of order four in $K$. To conclude with, substituting the results in (56) and (57) into (33), we may approximate the mean square error at sample size $K$ by

$$
\operatorname{MSE}_{K} \cong \frac{1}{4 \pi^{2}} \frac{\mathcal{P}^{(\leq 3)}(K)}{\mathcal{Q}^{(4)}(K)}
$$

$\mathcal{Q}^{(4)}$ being a polynomial of degree four, while the polynomial $\mathcal{P}^{(\leq 3)}$ is of degree at most three. The latter implies that MSE decreases at rate $1 / K$ for large $K$. 


\section{REFERENCES}

[1] Z. Wang and G. B. Giannakis, "Wireless multicarrier communications," IEEE Signal Processing Magazine, vol. 17, pp. 29-48, May 2000

[2] R. van Nee and R. Prasad, "OFDM for wireless multimedia communications," Artech House, 2000.

[3] T. Pollet and M. Moeneclaey, "The effect of carrier frequency offset on the performance of band limited single carrier and OFDM signals," in Proc. of IEEE Global Telecommunications Conference, vol. 1, pp. 719-723, Nov. 1996.

[4] H. Nishookar and R. Prasad, "On the sensitivity of multicarrier transmission over multipath channels to phase noise and frequency offset," in Proc. IEEE International Symposium on Personal, Indoor and Mobile Radio Communications, PIMRC, vol. 1, pp. 6872, Oct. 1996.

[5] Y. Zhao and S. Haggman, "Sensitivity to doppler shift and carrier frequency errors in OFDM systems - the consequences and solutions," in Proc. of the IEEE Vehicular Technology Conference, vol. 3, pp. 1564-1568, May 1996.

[6] S. Park, B. Seo, and C. Kang, "Effects of frequency offset compensation error on channel estimation for OFDM system under mobile radio channels," Signal Processing, vol. 83, pp. 2621-2630, Dec. 2003.

[7] M. Morelli and U. Mengali, "An improved frequency offset estimator for OFDM applications," IEEE Communications Letters, vol. 3 , pp. 75-77, Mar. 1999.

[8] M. Oh, X. Ma, G. Giannakis, and D. Park, "Hopping pilots for estimation of frequency-offset and multi-antenna channels in MIMO OFDM," in Proc. of the Global Telecommunications Conference, vol. 2, pp. 1084-1088, Dec. 2003.

[9] J. Yu and Y. Su, "Pilot-assisted maximum-likelihood frequencyoffset estimation for OFDM systems," IEEE Transactions on Communications, vol. 52, pp. 1997-2008, Nov. 2004.

[10] J. Zhang, G. Liao, and J. Wang, "Efficient frequency offset resolution for OFDM systems based on ML principle," IEEE Transactions on Consumer Electronics, vol. 50, pp. 799-806, Aug. 2004.

[11] P. Moose, "A technique for orthogonal frequency division multiplexing frequency offset correction," IEEE Transactions on Communications, vol. 42, pp. 2908-2914, Oct. 1994.

[12] M. Ghogho and A. Swami, "Semi-blind frequency offset synchronization for OFDM," in Proc. of the IEEE International Conference on Acoustics, Speech, and Signal Processing, vol. 3, pp. III-2333III-2336, May 2002.

[13] T. Roman, M. Enescu, and V. Koivunen, "Joint time-domain tracking of channel and frequency offsets for MIMO OFDM systems," Kluwer Wireless Personnal Communications, vol. 31, pp. 181-200, Dec. 2004.

[14] T. Schmidl and D. Cox, "Robust frequency and timing synchronization for OFDM," IEEE Transactions on Communications, vol. 45, pp. 1613-1621, Dec. 1997.

[15] U. Tureli, H. Liu, and M. Zoltowski, "OFDM blind carrier offset estimation: ESPRIT," IEEE Transactions on Communications, vol. 48, pp. 1459-1461, Sept. 2000.

[16] X. Ma, C. Tepedelenlioglu, G. Giannakis, and S. Barbarossa, "Nondata-aided carrier offset estimators for OFDM with null subcarriers: identifiability, algorithms, and performance," IEEE Journal on Selected Areas in Communications, vol. 19, pp. 2504-2515, Dec. 2001.

[17] H. Ge and K. Wang, "Efficient method for carrier offset correction in OFDM system," in Proc. of the IEEE International Conference on Acoustics, Speech, and Signal Processing, vol. 5, pp. 2467 2470, Mar. 1999

[18] M. Ghogho, A. Swami, and G. Giannakis, "Optimized nullsubcarrier selection for CFO estimation in OFDM over frequencyselective fading channels," in Proc. of the IEEE Global Telecommunications Conference, vol. 1, pp. 202-206, Nov. 2001.

[19] M. Ghogho and A. Swami, "Carrier frequency synchronization for OFDM systems," in Signal Processing for mobile communications handbook (M. Ibnkahla, ed.), ch. 8, CRC Press, 2005.

[20] J. van de Beek, M. Sandell, and P. Börjesson, "Ml estimation of time and frequency offset in OFDM systems," IEEE Transactions on Signal Processing, vol. 45, pp. 1800-1805, Jul. 1997.
[21] H. Bölcskei, "Blind high-resolution uplink synchronization of OFDM-based multiple access schemes," in Proc. of IEEE Workshop on Signal Processing Advances in Wireless Communications, pp. 166-169, May 1999.

[22] E. Chiavaccini and G. Vitetta, "Maximum-likelihood frequency recovery for OFDM signals transmitted over multipath fading channels," IEEE Transactions on Communications, vol. 52, pp. 244251, Feb. 2004.

[23] P. Ciblat and L. Vandendorpe, "Blind carrier frequency offset estimation for noncircular constellation-based transmissions," IEEE Transactions on Signal Processing, vol. 51, pp. 1378-1389, May 2003.

[24] P. Ciblat and M. Ghogho, "Harmonic retrieval in non-circular complex-valued multiplicative noise: Cramér-Rao bound," in Proc. of the IEEE International Conference on Acoustics, Speech, and Signal Processing, vol. 2, pp. II 489-II 492, May 2004.

[25] M. Ghogho and A. Swami, "Blind frequency-offset estimator for OFDM systems transmitting constant-modulus symbols," IEEE Communications Letters, vol. 6, pp. 343-345, Aug. 2002.

[26] T. Roman and V. Koivunen, "One-shot subspace based method for blind CFO estimation for OFDM," in Proc. of the IEEE International Conference on Acoustics, Speech, and Signal Processing, vol. 3, pp. 809-812, Mar. 2005.

[27] Y. Yao and G. Giannakis, "Blind carrier frequency offset estimation in SISO, MIMO, and multiuser OFDM systems," IEEE Transactions on Communications, vol. 53, pp. 173 - 183, Jan. 2005.

[28] H. Bölcskei, "Blind estimation of symbol timing and carrier frequency offset in wireless OFDM systems," IEEE Transactions on Communications, vol. 49, pp. 988-999, Jun. 2001.

[29] T. Roman and V. Koivunen, "Blind CFO estimation in OFDM systems using diagonality criterion," in Proc. of the IEEE International Conference on Acoustics, Speech, and Signal Processing, vol. 4, pp. IV 369-IV 372, May 2004.

[30] B. McNair, L. Cimini, and N. Sollenberger, "A robust timing and frequency offset estimation scheme for orthogonal frequency division multiplexing (OFDM) systems," in Proc. of the IEEE Vehicular Technology Conference, vol. 1, pp. 690-694, May 1999.

[31] X. Ma, H. Kobayashi, and S. Schwartz, "Joint frequency offset and channel estimation for OFDM," in Proc. of IEEE Global Telecommunications Conference, vol. 1, pp. 15-19, Dec. 2003.

[32] F. Neeser and J. Massey, "Proper complex random processes with applications to information theory," IEEE Transactions on Information Theory, vol. 39, pp. 1293-1302, Jul. 1993.

[33] B. Picinbono, "Second-order complex random vectors and normal distributions," IEEE Transactions on Signal Processing, vol. 44, pp. 2637-2640, Oct. 1996.

[34] J.Lei and T. Ng, "A blind carrier frequency offset detector used for feedback frequency synchronization in OFDM receivers," in Proc. of IEEE International Conference on Communications, vol. 3, pp. 2041-2045, May 2003.

[35] R. J. Serfling, Approximation Theorems of Mathematical Statistics. Wiley, 1980.

[36] R. Zhang, T. Tjhung, H. Hu, and P. He, "Window function and interpolation algorithm for ofdm frequency-offset correction," IEEE Transactions on Vehicular Technology, vol. 52, pp. 654-670, May 2003.

[37] S. Kai and E. Serpedin, "Coarse frame and carrier synchronization of OFDM systems: a new metric and comparison," IEEE Transactions on Wireless Communications, vol. 3, pp. 1271 - 1284, Jul. 2004.

[38] T. Roman, S. Visuri, and V. Koivunen, "Performance bound for blind CFO estimation in OFDM with real-valued constellations," in Proc. of the IEEE Semiannual Vehicular Technology Fall Conference, vol. 6, pp. 3866-3870, Sept. 2004.

[39] T. Roman, S. Visuri, and V. Koivunen, "Blind frequency synchronization for OFDM with real valued modulations," Technical Report 51, ISBN 951-22-7528-7, Helsinki University of Technology, Signal Processing Laboratory, Jan. 2005. Available at http://wooster.hut.fi/ troman/publications.

[40] J. Heiskala and J. Terry, OFDM Wireless LANs: A theoretical and practical guide. SAMS Publishing, 2001. 


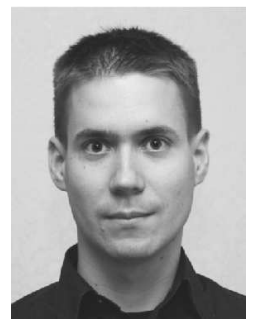

Timo Roman received his Engineer degree (Dipl. Eng.) in communication systems from the Swiss Federal Institute of Technology (EPFL), Switzerland, in July 2000. Part of his undergraduate studies was conducted at the Eurecom Institute, Sophia-Antipolis, France. From October 2000 to July 2001, he followed a one year post-graduate course program at EPFL while working as a part-time research engineer at STMicroelectronics, Geneva, Switzerland. Since fall 2001, Timo Roman is with the Signal Processing Laboratory, Helsinki University of Technology, Finland, pursuing his doctoral studies. His research interests include statistical signal processing in wireless communications oriented toward transceiver algorithm design for multi-carrier multiple-input multipleoutput (MIMO) systems in dispersive communication channels.

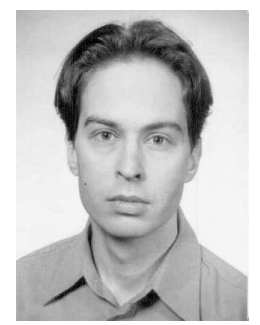

Samuli Visuri received the M.Sc, degree in Statistics from the University of Oulu, Finland in 1998 and the D.Sc. degree in Electrical Engineering from the Helsinki University of Technology, Finland in 2001. From 2002 to 2003 he worked as a post-doctoral researcher in Institut Eurecom, France (year 2002), and Swiss Federal Institute of Technology (ETH), Switzerland (year 2003). Since 2004 he has been working as a Senior Research Engineer at Nokia Research Center (Radio Technologies laboratory) Helsinki, Finland. His research interests include statistical signal processing and information theory with special emphasis on wireless communications, multi-input multi-output (MIMO) antenna systems, and Orthogonal Frequency Division Multiplexing (OFDM).

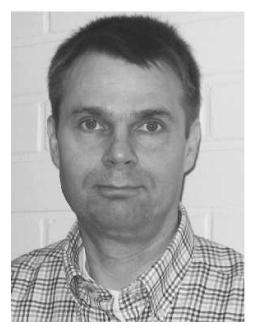

Visa Koivunen (Senior Member, IEEE) received his D.Sc. (Tech) degree with honors from the University of Oulu, Dept. of Electrical Engineering. From 1992 to 1995 he was a visiting researcher at the University of Pennsylvania, Philadelphia, USA. Year 1996 he held a faculty position at the Department of Electrical Engineering, University of Oulu. From August 1997 to August 1999 he was an Associate Professor at the Signal Processing Labroratory, Tampere University of Technology. Since 1999 he has been a Professor of Signal Processing at the Department of Electrical and Communications Engineering, Helsinki University of Technology (HUT), Finland. He is one of the Principal Investigators in SMARAD Center of Excellence in Radio and Communications Engineering nominated by the Academy of Finland. Since year 2003 he has been also adjunct professor at the University of Pennsylvania, Philadelphia, USA.

Dr. Koivunen's research interest include statistical, communications and sensor array signal processing. He has published more than 170 papers in international scientific conferences and journals. He has served as an associate editor for IEEE Signal Processing Letters. He is a member of the editorial board for the Signal Processing journal. He is also a member of the IEEE Signal Processing for Communication Technical Committee (SPCOM-TC). 